\title{
An abstract semilinear first order differential equation in the hyperbolic case
}

\author{
by JAN BOCHENEK (Kraków)
}

Abstract. This paper is devoted to the investigation of the abstract semilinear initial value problem

$$
d u / d t=A(t) u+f(t, u), \quad u(0)=u_{0},
$$

in the "hyperbolic" case.

1. Introduction. Let $X$ be a real reflexive Banach space and for each $t \in[0, T]$ let $A(t): X \rightarrow X$ be a linear closed densely defined operator. Let $u$ be a function from $\mathbb{R}$ into $X, f$ be a nonlinear function from $\mathbb{R} \times X$ into $X$ and $u_{0} \in X$. We consider the abstract semilinear initial value problem

$$
\left\{\begin{array}{l}
d u / d t=A(t) u+f(t, u), \quad t \in(0, T], \\
u(0)=u_{0} .
\end{array}\right.
$$

Our purpose is to prove the theorem on the existence and uniqueness of the solution of the problem (1). We shall use the existence and uniqueness theorem for the linear problem corresponding to (1). In Section 3 we formulate and prove a theorem on the existence and uniqueness for the linear problem which is the same as Theorem 1 of [3] but the proof is slightly simpler.

2. Preliminaries. Let $\{A(t)\}, t \in[0, T]$, be a family of operators as in Section 1. We make the following assumptions on $\{A(t)\}$.

$\left(\mathrm{Z}_{1}\right)$ For each $t \in[0, T], A(t)$ is the generator of a $C_{0}$-semigroup, the domains $D(A(t))=D$ do not depend on $t$ and $D$ is dense in $X$.

1991 Mathematics Subject Classification: 34K30, 34G20.

Key words and phrases: semilinear initial value problem, stable family of operators, hyperbolic case.

This paper was supported by KBN, grant 211189101. 
$\left(\mathrm{Z}_{2}\right)$ The family $\{A(t)\}, t \in[0, T]$, is stable in the sense that there exist real numbers $M \geq 1$ and $\omega$ such that

$$
\left\|\prod_{j=1}^{k}\left(A\left(t_{j}\right)-\lambda\right)^{-1}\right\| \leq M(\lambda-\omega)^{-k}
$$

for all $\lambda>\omega, 0 \leq t_{1} \leq \ldots \leq t_{k} \leq T, k \in \mathbb{N}$.

$\left(\mathrm{Z}_{3}\right)$ For each $x \in X$ the mapping $t \rightarrow A(t) x$ is of class $C^{1}$ on $[0, T]$.

$\left(\mathrm{Z}_{4}\right)$ For each $t \in[0, T], 0$ belongs to the resolvent set of $A(t)$.

The set of conditions $\left(\mathrm{Z}_{1}\right)-\left(\mathrm{Z}_{3}\right)$ is usually referred to as the "hyperbolic" case in contrast to the "parabolic" case where each $A(t), t \geq 0$, is assumed to be the infinitesimal generator of an analytic semigroup (cf. [4, Chapter 5]). This terminology comes from different applications of the abstract results to partial differential equations.

Under assumptions $\left(\mathrm{Z}_{1}\right)-\left(\mathrm{Z}_{3}\right)$ there exists a unique function $U$, called the fundamental solution of the problem (1), defined on the triangle $\Delta_{T}:=$ $\{(t, s): 0 \leq s \leq t \leq T\}$ and having the following properties:

(a) $U(t, s) \in B(X)$ and $\|U(t, s)\| \leq M e^{\omega(t-s)}$,

(b) $U(t, s)$ is strongly continuous on $\Delta_{T}, U(t, t)=I$ and $U(t, r) U(r, s)=$ $U(t, s)$ for $0 \leq s \leq r \leq t \leq T$,

(c) $U(t, s)$ maps $D$ into $D$,

(d) for each $x \in D, U(t, s) x$ is continuously differentiable on $\Delta_{T}$ and

$$
\frac{\partial}{\partial t} U(t, s) x=A(t) U(t, s) x, \quad \frac{\partial}{\partial s} U(t, s) x=-U(t, s) A(s) x .
$$

Lemma 1. Under assumptions $\left(\mathrm{Z}_{1}\right)-\left(\mathrm{Z}_{4}\right)$ for each $x \in X$ and $\alpha, \beta \in[0, t]$,

$$
\int_{\alpha}^{\beta} U(t, s) x d s \in D \quad \text { and } \quad A(t) \int_{\alpha}^{\beta} U(t, s) x d s=[H(t, \beta)-H(t, \alpha)] x,
$$

where $H(t, s) \in B(X)$ and $H$ is strongly continuous on $\Delta_{T}$.

Proof. The proof starts with the observation that

$$
\begin{aligned}
\int_{\alpha}^{\beta} U(t, s) x d s= & \int_{\alpha}^{\beta} U(t, s) A(s) A^{-1}(s) x d s=-\int_{\alpha}^{\beta} \frac{\partial}{\partial s} U(t, s)\left(A^{-1}(s) x\right) d s \\
= & -\left.U(t, s)\left(A^{-1}(s) x\right)\right|_{\alpha} ^{\beta}+\int_{\alpha}^{\beta} U(t, s) \frac{d}{d s}\left(A^{-1}(s) x\right) d s \\
= & -\left.U(t, s)\left(A^{-1}(s) x\right)\right|_{\alpha} ^{\beta} \\
& +\int_{\alpha}^{\beta} U(t, s) A^{-1}(s) \frac{d}{d s}(A(s)) A^{-1}(s) x d s .
\end{aligned}
$$


From this, using property (c) of $U(t, s)$ and the closedness of $A(t)$, we have

$$
\int_{\alpha}^{\beta} U(t, s) x d s \in D
$$

Therefore we get

$$
\begin{aligned}
A(t) \int_{\alpha}^{\beta} U(t, s) x d s= & -\left.A(t) U(t, s)\left(A^{-1}(s) x\right)\right|_{\alpha} ^{\beta} \\
& +\int_{\alpha}^{\beta} A(t) U(t, s) A^{-1}(s) \frac{d}{d s}(A(s)) A^{-1}(s) x d s .
\end{aligned}
$$

Define

$$
H(t, s):=\int_{0}^{s} A(t) U(t, \tau) A^{-1}(\tau) \frac{d}{d \tau}(A(\tau)) A^{-1}(\tau) d \tau-A(t) U(t, s) A^{-1}(s)
$$

for $0 \leq s \leq t \leq T$. Since $A(t) U(t, s) A^{-1}(s)$ and $\frac{d}{d s}(A(s)) A^{-1}(s)$ are bounded and strongly continuous operators on $\Delta_{T}$, this finishes the proof.

Lemma 2 ([3], Lemma 3). Let $h: \Delta_{T} \rightarrow X$ and suppose that:

(i) for almost all $s \in[0, t]$ the function $[0, T] \ni t \rightarrow h(t, s)$ is continuous,

(ii) for each $t \in[0, T], h(t, \cdot)$ is summable over $[0, t]$,

(iii) there exists $N>0$ such that for $(t, s) \in \Delta_{T},\|h(t, s)\| \leq N$.

Then the function $G:[0, T] \rightarrow X$ defined as

$$
G(t):=\int_{0}^{t} h(t, s) d s
$$

is continuous.

LEMMA 3. Suppose that:

(i) assumptions $\left(\mathrm{Z}_{1}\right)-\left(\mathrm{Z}_{4}\right)$ hold,

(ii) a function $g:[0, T] \rightarrow X$ satisfies the Lipschitz condition with constant $L>0$.

Then for every fixed $t_{0} \in[0, T)$ the formula

$$
v(t)=\int_{t_{0}}^{t} U(t, s) g(s) d s, \quad t \in\left(t_{0}, T\right]
$$

defines a function from $\left[t_{0}, T\right]$ into $D$, the function $w:\left[t_{0}, T\right] \rightarrow X$ given by

$$
w(t):=A(t) \int_{t_{0}}^{t} U(t, s) g(s) d s=A(t) v(t)
$$


is continuous and

(6) $\left\|A(t) \int_{t_{0}}^{t} U(t, s) g(s) d s\right\|$

$$
\leq\left\|\left[H(t, t)-H\left(t, t_{0}\right)\right] g\left(t_{0}\right)\right\|+M L\left(t-t_{0}\right), \quad t \in\left[t_{0}, T\right] .
$$

Proof (cf. [1], proof of Lemma 1 ). Let $t \in\left[t_{0}, T\right]$. For every $n \in \mathbb{N}$ set

$$
u_{n}(t):=\int_{t_{0}}^{t} U(t, s) g_{n}(s) d s
$$

where

$$
g_{n}(s):=\sum_{i=0}^{n-1} g\left(t_{i}\right) \chi_{B_{i}}(s), \quad s \in\left[t_{0}, t\right] ;
$$

here $B_{i}=\left[t_{i}, t_{i+1}\right)$ for $i=0,1, \ldots, n-2, B_{n-1}=\left[t_{n-1}, t_{n}\right], t_{i}=t_{0}+$ $(i / n)\left(t-t_{0}\right), i=0,1, \ldots, n$, and $\chi_{B_{i}}$ denotes the characteristic function of $B_{i}$.

Clearly $\left\{g_{n}\right\}$ converges uniformly on $\left[t_{0}, t\right]$ to $g$ (in the norm topology) and $\left\{u_{n}(t)\right\}$ converges in norm to $v(t)$. By Lemma 1 , for each $x \in X$ and for $\alpha, \beta \in[0, t]$, we have

$$
\int_{\alpha}^{\beta} U(t, s) x d s \in D
$$

and

$$
A(t) \int_{\alpha}^{\beta} U(t, s) x d s=[H(t, \beta)-H(t, \alpha)] x,
$$

where $H(t, s) \in B(X)$ and $H$ is strongly continuous on $\Delta_{T}$.

By $(7)-(9),\left\{u_{n}(t)\right\} \subset D$. By (10) we have

$$
\begin{aligned}
A(t) u_{n}(t)= & \sum_{i=0}^{n-1}\left[H\left(t, t_{i+1}\right)-H\left(t, t_{i}\right)\right] g\left(t_{i}\right) \\
= & {\left[H(t, t)-H\left(t, t_{0}\right)\right] g\left(t_{0}\right)+H\left(t, t_{1}\right)\left[g\left(t_{0}\right)-g\left(t_{1}\right)\right] } \\
& +\ldots+H\left(t, t_{n-1}\right)\left[g\left(t_{n-2}\right)-g\left(t_{n-1}\right)\right] \\
& +H(t, t)\left[g\left(t_{n-1}\right)-g\left(t_{0}\right)\right] .
\end{aligned}
$$

Now the Lipschitz condition for $g$ gives

(11) $\quad\left\|A(t) u_{n}(t)\right\| \leq\left\|\left[H(t, t)-H\left(t, t_{0}\right)\right] g\left(t_{0}\right)\right\|+M L\left(t-t_{0}\right)$,

where $M:=2 \sup \{\|H(t, s)\|: 0 \leq s \leq t \leq T\}$. On the other hand, for every $x^{*} \in D\left(A^{*}(t)\right)$ we have

(12) $\left\langle A(t) u_{n}(t), x^{*}\right\rangle=\left\langle u_{n}(t), A^{*}(t) x^{*}\right\rangle \rightarrow\left\langle v(t), A^{*}(t) x^{*}\right\rangle$. 
Since $D\left(A^{*}(t)\right)$ is dense in $X^{*}$, from (11) and (12) it follows that $\left\{A(t) u_{n}(t)\right\}$ is weakly convergent in $X$. Since $A(t)$ is closed, it follows that $v(t) \in D=$ $D(A(t))$ and that the inequality (6) holds.

To prove the continuity of the function $w$, we first recall that if $g$ : $[0, T] \rightarrow X$ satisfies the Lipschitz condition and $X$ is a reflexive Banach space then $g^{\prime}(t)$ exists for almost every $t \in[0, T], g^{\prime}$ is integrable over every $[\alpha, \beta] \subset[0, T]$ and

$$
\int_{\alpha}^{\beta} g^{\prime}(s) d s=g(\beta)-g(\alpha)
$$

Hence

$$
g(s)=g\left(t_{0}\right)+\int_{t_{0}}^{s} g^{\prime}(\tau) d \tau \quad \text { for } s \in\left[t_{0}, T\right] .
$$

Now we can rewrite (4) as

$$
\begin{aligned}
v(t) & =\int_{t_{0}}^{t} U(t, s)\left[g\left(t_{0}\right)+\int_{t_{0}}^{s} g^{\prime}(\tau) d \tau\right] d s \\
& =\int_{t_{0}}^{t} U(t, s) g\left(t_{0}\right) d s+\int_{t_{0}}^{t}\left[\int_{\tau}^{t} U(t, s) g^{\prime}(\tau) d s\right] d \tau .
\end{aligned}
$$

Since each term on the right-hand side belongs to $D$, application of Lemma 1 enables us to write

$$
\begin{aligned}
w(t)= & A(t) v(t) \\
& =H(t, t) g\left(t_{0}\right)-H\left(t, t_{0}\right) g\left(t_{0}\right)+\int_{t_{0}}^{t}\left[H(t, t) g^{\prime}(\tau)-H(t, \tau) g^{\prime}(\tau)\right] d \tau \\
& =H(t, t) g(t)-H\left(t, t_{0}\right) g\left(t_{0}\right)-\int_{t_{0}}^{t} H(t, \tau) g^{\prime}(\tau) d \tau .
\end{aligned}
$$

Now the continuity of $w$ follows directly by Lemmas 1 and 2 . This completes the proof.

3. Linear inhomogeneous problem corresponding to (1). In this section we consider the linear problem corresponding to (1), i.e.

$$
\left\{\begin{array}{l}
d u / d t=A(t) u+g(t), \quad t \in(0, T], \\
u(0)=u_{0} .
\end{array}\right.
$$

Definition 1. A function $u:[0, T] \rightarrow X$ is said to be a (classical) solution of (14) if $u$ is continuous on $[0, T]$, continuously differentiable on $(0, T), u(t) \in D(A(t))$ for $0 \leq t \leq T$ and (14) is satisfied. 
THEOREM 1. Let assumptions $\left(\mathrm{Z}_{1}\right)-\left(\mathrm{Z}_{4}\right)$ hold. Assume g satisfies the Lipschitz condition in $[0, T]$ and $u_{0} \in D$. Then the problem (14) has exactly one solution $u$, given by

$$
u(t)=U(t, 0) u_{0}+\int_{0}^{t} U(t, s) g(s) d s
$$

Proof. Since the function $\widetilde{u}(t)=U(t, 0) u_{0}$ is the solution of the corresponding homogeneous equation with initial condition $\widetilde{u}(0)=u_{0}$, we need only show that the function $v$ defined by $(4)$, with $t_{0}=0$, satisfies the equation in (14) and the condition $v(0)=0$. Let $t \in(0, T]$ be fixed and let $h>0$ be such that $(t+h) \in(0, T]$. We have

$$
v(t+h)-v(t)=\int_{0}^{t}[U(t+h, s)-U(t, s)] g(s) d s+\int_{t}^{t+h} U(t+h, s) g(s) d s .
$$

Therefore

$$
h^{-1}[v(t+h)-v(t)]=h^{-1}[U(t+h, t)-I] v(t)+h^{-1} \int_{t}^{t+h} U(t+h, s) g(s) d s .
$$

Since $v(t) \in D$, we get

$$
\lim _{h \rightarrow 0} h^{-1}[U(t+h, t)-I] v(t)=A(t) v(t) .
$$

From the inequality

$$
\begin{aligned}
& \left\|h^{-1} \int_{t}^{t+h} U(t+h, s) g(s) d s-g(t)\right\| \\
& \leq\left\|h^{-1} \int_{t}^{t+h} U(t+h, s)[g(s)-g(t)] d s\right\|+\left\|h^{-1} \int_{t}^{t+h}[U(t+h, s)-I] g(t) d s\right\| \\
& \leq C L h^{-1} \int_{t}^{t+h}(s-t) d s+\sup \{\|[U(t+h, s)-I] g(t)\|: s \in[t, t+h]\},
\end{aligned}
$$

where $C:=\sup \left\{\|U(t, s)\|:(t, s) \in \Delta_{T}\right\}$ and $L$ is the Lipschitz constant for $g$, it follows immediately that

$$
\lim _{h \rightarrow 0} h^{-1} \int_{t}^{t+h} U(t+h, s) g(s) d s=g(t) .
$$

By (16) and (17) we have

$$
\left(D^{+} v\right)(t)=A(t) v(t)+g(t) \quad \text { for } t \in(0, T],
$$

where $D^{+}$means the right derivative. From (12) it follows by Lemma 4 that $D^{+} v$ is a continuous function in $(0, T)$. Hence the derivative $d v / d t$ exists 
and

$$
\frac{d v}{d t}(t)=\left(D^{+} v\right)(t)=A(t) v(t)+g(t) \quad \text { for } t \in(0, T]
$$

It is obvious that $v(0)=0$. This proves that $u$ given by (15) is a solution of (14).

To show the uniqueness it suffices to remark that if $U(t, s)$ is a fundamental solution of (14), $g:[0, T] \rightarrow X$ is continuous and $u$ is a solution of (14), then $u$ is given by (15) (cf. [5, Theorem 4.5.1]). This completes the proof.

4. Existence and uniqueness of solution of problem (1). In this section we consider the nonlinear problem (1), mentioned in the introduction. Suppose that the family $\{A(t)\}$ satisfies the same assumptions as in Section 2 and let $f:[0, T] \times X \rightarrow X$ be continuous.

The solution of the problem (1) is defined analogously to the solution of (14) (cf. Def. 1).

Similarly to the linear case we have the following

THEOREM 2. If $f:[0, T] \times X \rightarrow X$ is continuous and $u$ is a solution of the problem (1), then u satisfies the integral equation

$$
u(t)=U(t, 0) u_{0}+\int_{0}^{t} U(t, s) f(s, u(s)) d s,
$$

where $U(t, s)$ is the fundamental solution of (1) defined in Section 2.

TheOREM 3. Let assumptions $\left(\mathrm{Z}_{1}\right)-\left(\mathrm{Z}_{4}\right)$ hold and let $f:[0, T] \times X \rightarrow X$ be continuous. Suppose that there exists $L>0$ such that

$$
\|f(t, x)-f(t, y)\| \leq L\|x-y\| \quad \text { for } t \in[0, T], x, y \in X .
$$

Then for any $u_{0} \in X$ there exists exactly one continuous solution of (19) in $[0, T]$.

Pr o of. Let $C:=\sup \left\{\|U(t, s)\|:(t, s) \in \Delta_{T}\right\}$. In the space $C([0, T], X)$ consider the two norms

$$
\begin{aligned}
\|u\| & :=\sup \{\|u(t)\|: t \in[0, T]\}, \\
|u| & :=\sup \left\{e^{-C L T}\|u(t)\|: t \in[0, T]\right\}
\end{aligned}
$$

(cf. [6, Theorem 4.5]). Since $|u| \leq\|u\| \leq e^{C L T}|u|$, these norms are equivalent. Let

$$
(G u)(t):=U(t, 0) u_{0}+\int_{0}^{t} U(t, s) f(s, u(s)) d s, \quad t \in[0, T] .
$$

Analogously to the proof of Theorem 4.5 of [6] we can prove that (22) defines an operator from $C([0, T], X)$ into itself and that $G$ is a contraction under 
the norm (21). By Banach's contraction principle, this implies that $G$ has a unique fixed point $u$, and $G^{n}(y) \rightarrow u$ for each $y \in C([0, T], X)$, where $G^{0}(y):=y$ and $G^{n+1}(y):=G\left(G^{n}(y)\right), n=0,1,2, \ldots$ (see [2, Chapter I]). Theorem 3 is proved.

Theorem 4. Assume $\left(\mathrm{Z}_{1}\right)-\left(\mathrm{Z}_{4}\right)$ and let $u_{0} \in D$. Let $f:[0, T] \times X \rightarrow X$ be a Lipschitzian mapping with Lipschitz constant $L>0$. Then the problem (1) has a unique solution which is a solution of the integral equation (19).

The proof of this theorem is based on the following lemmas.

LEMMA 4. Suppose that:

(i) $f:[0, T] \times X \rightarrow X$ is continuous,

(ii) there exists $L>0$ such that $\|f(t, x)-f(t, y)\| \leq L\|x-y\|$ for $t \in[0, T], x, y \in X$,

(iii) $u_{n}:[0, T] \rightarrow X$ is continuous for each $n \in \mathbb{N}$,

(iv) $\left\{u_{n}\right\}$ is uniformly convergent in $[0, T]$.

Then there is a constant $C>0$ such that

$$
\left\|f\left(t, u_{n}(t)\right)\right\| \leq C \quad \text { for } t \in[0, T] \text { and } n \in \mathbb{N} .
$$

Proof. Let $u:=\lim _{n \rightarrow \infty} u_{n}$. From (iii) and (iv) we see that $u:[0, T] \rightarrow$ $X$ is a continuous function. By (i), the mapping $\widetilde{f}:[0, T] \rightarrow X$, where $\widetilde{f}(t):=f(t, u(t))$, is continuous, and hence bounded. On the other hand, by (ii), the sequence $\left\{\widetilde{f}_{n}\right\}$ where $\widetilde{f}_{n}(t):=f\left(t, u_{n}(t)\right)$ for $t \in[0, T]$, uniformly converges to $\tilde{f}$ on $[0, T]$. This finishes the proof.

LEMMA 5. Under the assumptions of Theorem 4 the solution $u$ of the equation (19) satisfies the Lipschitz condition on $[0, T]$.

Proof. From Theorem 3 it follows that (19) has a unique solution $u \in$ $C([0, T], X)$ such that

$$
u=\lim _{n \rightarrow \infty} u_{n}
$$

where

$$
\left\{\begin{array}{l}
u_{n+1}(t)=U(t, 0) u_{0}+\int_{0}^{t} U(t, s) f\left(s, u_{n}(s)\right) d s, \\
u_{0}(t):=u_{0},
\end{array}\right.
$$

for $n=0,1,2, \ldots, t \in[0, T]$. The convergence in (23) is uniform on $[0, T]$.

We first prove by induction that each $u_{n}:[0, T] \rightarrow X$ satisfies the Lipschitz condition. For $n=0$ this is obvious. Assume that $u_{0}, u_{1}, \ldots, u_{n}$ satisfy the Lipschitz condition in $[0, T]$. 
Since $f$ satisfies the Lipschitz condition, so do the mappings $[0, T] \ni t \rightarrow$ $f\left(t, u_{k}(t)\right)$ for $k=0,1, \ldots, n$. By Theorem 1 , from (24) we get

$$
\frac{d u_{n+1}}{d t}=A(t) u_{n+1}(t)+f\left(t, u_{n}(t)\right), \quad t \in(0, T] .
$$

Since the right side of $(25)$ is a continuous function in $[0, T]$, we have

$$
L_{n+1}:=\sup \left\{\left\|d u_{n+1} / d t\right\|: t \in[0, T]\right\}<\infty .
$$

It is clear that $u_{n+1}$ satisfies the Lipschitz condition with constant $L_{n+1}$. Therefore each $u_{n}, n=0,1, \ldots$, satisfies the Lipschitz condition with constant $L_{n}, n=0,1, \ldots$

Now we prove that the sequence $\left\{L_{n}\right\}$ is bounded from above. From (25) and (24), by (6), we obtain

$$
\begin{aligned}
\left\|\frac{d u_{n+1}}{d t}\right\| & \leq\left\|A(t) u_{n+1}(t)\right\|+\left\|f\left(t, u_{n}(t)\right)\right\| \\
& \leq\left\|A(t) U(t, 0) u_{0}\right\|+\left\|A(t) \int_{0}^{t} U(t, s) f\left(s, u_{n}(s)\right) d s\right\|+C \\
& \leq C_{1}+C+\left\|[H(t, t)-H(t, 0)] f\left(0, u_{0}\right)\right\|+M L\left(1+L_{n}\right) t \\
& \leq C_{1}+C+M C+M L T+M L L_{n} t=K^{(1)}+M L L_{n} t
\end{aligned}
$$

where $C:=\sup \left\{\left\|f\left(t, u_{n}(t)\right)\right\|: t \in[0, T], n \in \mathbb{N}\right\}, C_{1}:=\sup \left\{\left\|A(t) U(t, 0) u_{0}\right\|:\right.$ $t \in[0, T]\}, M:=2 \sup \{\|H(t, s)\|: 0 \leq s \leq t \leq T\}$ and $K^{(1)}:=C_{1}+C+$ $M(C+L T)$.

Let $t \in[0,1 /(2 M L)]$. Then from the above estimate we get

$$
L_{n+1}^{(1)} \leq K^{(1)}+\alpha L_{n}^{(1)},
$$

where

$$
L_{k}^{(1)}:=\sup \left\{\left\|d u_{k} / d t\right\|: t \in[0,1 /(2 M L)]\right\}, \quad k=0,1,2, \ldots,
$$

and $\alpha:=M L t \leq 1 / 2$.

The inequality (27) in turn implies

$$
\begin{aligned}
L_{n+1}^{(1)} & \leq K^{(1)}+\alpha L_{n}^{(1)} \leq K^{(1)}+\alpha\left(K^{(1)}+\alpha L_{n-1}^{(1)}\right) \leq \ldots \\
& \leq K^{(1)}\left(1+\alpha+\ldots+\alpha^{n}\right)+\alpha^{n+1} L_{0}^{(1)} \leq K^{(1)} \frac{1}{1-\alpha} \leq 2 K^{(1)}
\end{aligned}
$$

since $L_{0}^{(1)}=0$. This implies that

$$
L_{n}^{(1)} \leq 2 K^{(1)}, \quad n \in \mathbb{N} .
$$


Setting $t_{1}:=1 /(2 M L)$ we consider equation $(25)$ for $\left(t_{1}, 2 t_{1}\right]$. The solution of this equation is given by

$$
u_{n+1}(t)=U\left(t, t_{1}\right) u_{n+1}\left(t_{1}\right)+\int_{t_{1}}^{t} U(t, s) f\left(s, u_{n}(s)\right) d s, \quad t \in\left[t_{1}, 2 t_{1}\right] .
$$

As above we have

$$
\begin{aligned}
\left\|\frac{d u_{n+1}}{d t}\right\| \leq & \left\|A(t) u_{n+1}(t)\right\|+\left\|f\left(t, u_{n}(t)\right)\right\| \\
\leq & \left\|A(t) U\left(t, t_{1}\right) u_{n+1}\left(t_{1}\right)\right\|+\left\|A(t) \int_{t_{1}}^{t} U(t, s) f\left(t, u_{n}(s)\right) d s\right\|+C \\
\leq & \left\|A(t) U\left(t, t_{1}\right) A^{-1}\left(t_{1}\right)\right\|\left\|A\left(t_{1}\right) u_{n+1}\left(t_{1}\right)\right\| \\
& +\left\|\left[H(t, t)-H\left(t, t_{1}\right)\right] f\left(t_{1}, u_{n}\left(t_{1}\right)\right)\right\|+M L\left(1+L_{n}\right)\left(t-t_{1}\right)+C \\
\leq & C_{2}\left(2 K^{(1)}+C\right)+M C+M L T+C+M L L_{n}\left(t-t_{1}\right),
\end{aligned}
$$

where $C_{2}:=\sup \left\{\left\|A(t) U(t, s) A^{-1}(s)\right\|: 0 \leq s \leq t \leq T\right\}$ and

$$
\left\|A\left(t_{1}\right) u_{n+1}\left(t_{1}\right)\right\| \leq\left\|\frac{d u_{n+1}}{d t}\left(t_{1}\right)\right\|+\left\|f\left(t_{1}, u_{n}\left(t_{1}\right)\right)\right\| \leq 2 K^{(1)}+C .
$$

From this for $t \in\left[t_{1}, 2 t_{1}\right]$ we get

$$
L_{n+1}^{(2)} \leq K^{(2)}+\alpha L_{n}^{(2)}
$$

where

$$
\begin{gathered}
L_{k}^{(2)}:=\sup \left\{\left\|d u_{k} / d t\right\|: t \in\left[t_{1}, 2 t_{1}\right]\right\}, \quad k=0,1,2, \ldots, \\
\alpha:=M L\left(t-t_{1}\right) \leq 1 / 2, \quad K^{(2)}:=2 K^{(1)} C_{2}+C\left(C_{2}+M+1\right)+M L T .
\end{gathered}
$$

Inequality (29), just as above, implies the inequality

$$
L_{n}^{(2)} \leq 2 K^{(2)}, \quad n \in \mathbb{N} .
$$

Considering next the interval $\left[2 t_{1}, 3 t_{1}\right]$ and so on, after $l$ steps we cover the whole interval $[0, T]$, where $[2 L M T] \leq l<[2 L M T]+1$.

Let $K:=2 \max \left(K^{(1)}, \ldots, K^{(l)}\right)$. The above considerations lead to

$$
L_{n} \leq K, \quad n \in \mathbb{N} .
$$

Let now $t, t+h \in[0, T]$. We have

$$
\left\|u_{n}(t+h)-u_{n}(t)\right\| \leq L_{n}|h| \leq K|h|, \quad n \in \mathbb{N} .
$$

Thus, by letting $n \rightarrow \infty$, we see by (32) that

$$
\|u(t+h)-u(t)\| \leq K|h| .
$$

The proof is complete.

Proof of Theorem 4. By (33) and the assumption that $f$ is Lipschitzian, the mapping $[0, T] \ni t \rightarrow f(t, u(t))$ also satisfies the Lipschitz 
condition. Let $u \in C([0, T], X)$ be a solution of the integral equation (19). Then, by Theorem $1, u$ is a solution of the equation

$$
\frac{d z}{d t}=A(t) z+f(t, u(t)), \quad t \in(0, T],
$$

with the initial condition

$$
z(0)=u_{0} .
$$

This means that $u$ is a solution of the problem (1).

The uniqueness for the problem (1) follows immediately from the uniqueness of the solution of (19).

\section{References}

[1] J. Bochenek, An abstract nonlinear second order differential equation, Ann. Polon. Math. 54 (1991), 155-166.

[2] J. Dugundji and A. Granas, Fixed Point Theory, Vol. I, PWN, Warszawa, 1982.

[3] M. Kozak, An abstract nonlinear temporally inhomogeneous equation, Demonstratio Math. 23 (1990), 993-1003.

[4] A. Pazy, Semigroups of Linear Operators and Applications to Partial Differential Equations, Springer, 1983.

[5] H. Tanabe, Equations of Evolution, Pitman, London, 1979.

[6] T. Winiarska, Differential Equations with Parameter, Monograf. 68, Technical University of Cracow, 1988.

INSTITUTE OF MATHEMATICS

CRACOW UNIVERSITY OF TECHNOLOGY

WARSZAWSKA 24

31-155 KRAKÓW, POLAND 